\title{
The Effects Of Improvements In Information Technology On Growth Of Cities: An Extension
}

Alexandros Panayides, (E-mail: panayidesa@wpunj.edu), William Paterson University

\begin{abstract}
How will improvements in information technology affect growth of cities? Futurists have suggested that as telecommunications technology improves there will be no need for face-to-face contact and therefore cities, in which face-to-face interactions are facilitated, will become obsolete. This paper develops a general model where individuals involved in a relationship use two modes of interaction, face-to-face interactions and electronic communications. Comparative static analysis on the optimal solutions shows that improvements in telecommunications technology may increase or decrease the demand for face-to-face interactions, depending whether face-to-face interactions and telecommunications are substitutes or complements. The placement of the model within a spatial framework shows that city size increases with electronic communications if faceto-face interactions and electronic communications are complements.
\end{abstract}

\section{Introduction}

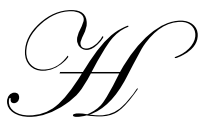

ow will advances in telecommunications technology affect location decisions and the size of cities? Futurists have argued that improvements in electronic communications will reduce the demand for face-to-face interactions, and therefore will make cities, in which face-to-face interactions are facilitated, obsolete. ${ }^{1}$ In an attempt to examine the interdependence between improvements in electronic communications and face-to-face interactions, Gaspar and Glaeser (1998) developed a general model where either telecommunications or face-to-face interactions are used in a relationship and show that advanced communications technology can either increase or decrease both face-to-face interactions and city size. Panayides (2001) using a specific quadratic function presented a model in which allow the simultaneous use of both modes of interaction, face-to-face and electronic communications, in a relationship. Panayides (2001) assumed that better electronic communications do not increase the payoff from face-to-face interactions, and second, that more valuable face-to-face interactions do not increase the payoff from electronic communications. In this paper we extend Panayides analysis by relaxing both of these assumptions.

\section{The Payoff Model}

In this section we construct a payoff model using a quadratic function to examine the effects of improvements in electronic communications on the time spent on face-to-face interactions. First, the quadratic function is so constructed that face-to-face interactions and electronic communications are substitutes (general model I), and then such that face-to-face interactions and electronic communications are complements (general model II).

General Model I (Face-to-face interactions and electronic communications are substitutes) ${ }^{2}$ : Consider a representative agent involved in relationships with other agents. The relationships are conducted using both electronic communications and face-to-face contact.

Readers with comments or questions are encouraged to contact the authors via email.

\footnotetext{
${ }^{1}$ Toffler (1980), Negroponte (1995), Naisbitt (1995), and Knoke (1996) are recent scholars who support this view and predict the end of cities.

${ }^{2}$ The structure of the general and urban models is similar to Panayides (2001).
} 
The payoff of the relationships depends on the time spent on electronic and face-to-face interactions and is given by the following function,

$$
V=a_{e} e^{t}+a_{f}^{b t} f-\frac{1}{2} a_{e e} t^{2}-\frac{1}{2} a_{f f} b^{2} t_{f}^{2}-a_{e} a_{f} a_{e f} e_{e}^{b t}{ }_{f}{ }^{3}
$$

where: $t_{e}$ is the time spent on electronic communications, $t_{f}$ is the time spent on face-to-face communications $b$ is the fraction of face-to-face communication that is productive, $a_{e}$ is the parameter that increases the productivity of electronic communications, and $a_{f}$ is the parameter that increases the value of face-to-face interactions. Note that $\frac{\partial^{2} V}{\partial t_{e} \partial b t_{f}}=-a_{e} a_{f} a_{e f}<0$, implying that face-to-face interactions and electronic communications are substitutes.

Both modes of interaction, face-to-face and electronic use a common input, time, which is available at cost c per unit. The total cost of engaging in interactions is then given by the following function,

$$
C=c\left(t_{e}+t_{f}\right)
$$

The individual is then faced with the problem of maximizing her net payoff from interactions, i.e., she is faced with the following problem:

$$
\operatorname{Max} \Pi=a_{e} t_{e}+a_{f} b t_{f}-\frac{1}{2} a_{e e} t_{e}^{2}-\frac{1}{2} a_{f f} b^{2} t_{f}^{2}-a_{e} a_{f} a_{e f} e^{b t_{f}}-c\left(t_{e}+t_{f}\right)
$$

The first order conditions (FOCs) for payoff maximization are

$$
\begin{aligned}
& \frac{\partial \Pi}{\partial t_{e}}=a_{e}-a_{e e} t_{e}-a_{e} a_{f} a_{e f} b t_{f}-c=0 \\
& \Rightarrow\left(1-a_{f} a_{e f} b t_{f}\right)=\frac{c+a_{e e} t_{e}}{a_{e}}, \\
& \frac{\partial \Pi}{\partial t_{f}}=b a_{f}-a_{f f} b^{2} t_{f}-a_{e} a_{f} a_{e f} b t_{e}-c=0 \\
& \Rightarrow\left(1-a_{e} a_{e f} t_{e}\right)=\frac{c+a_{f f} b^{2} t_{f}}{b a_{f}} .
\end{aligned}
$$

Both first order conditions imply that the marginal payoff from either mode of interaction must be equal with the marginal cost, i.e., the opportunity cost of time.

\footnotetext{
${ }^{3}$ The difference between the payoff function (1) and the payoff function in Panayides (2001) is that $a_{e}$ and $a_{f}$ affect both $t_{e}$ and $t_{f}$, which implies that if the two modes of interaction are substitutes, both partial derivatives $\frac{\partial^{2} V}{\partial t_{e} \partial a_{f}}$ and $\frac{\partial^{2} V}{\partial b t_{f} \partial a_{e}}$ are not zero anymore but negative.
} 
The second order conditions (SOCs) for a maximum are that the following Hessian determinant be positive:

$$
|H|=\left|\begin{array}{cc}
-a_{e e} & -b a_{e} a_{f} a_{e f} \\
-b a_{e} a_{f} a_{e f} & -b^{2} a_{f f}
\end{array}\right|=b^{2}\left(a_{e e} a_{f f}-\left(a_{e} a_{f} a_{e f}\right)^{2}\right)>0
$$

Using (3) and (4) the following optimal solutions for $t_{e}^{*}$ and $t_{f}^{*}$ are obtained:

$$
\begin{aligned}
& t_{f}^{*}=\frac{b a_{e e} a_{f}-b a_{e f} a_{f} a_{e}^{2}+b c a_{e f} a_{e} a_{f}-c a_{e e}}{|H|}, \\
& t_{e}^{*}=\frac{a_{e}-c}{a_{e e}}-\frac{b a_{e f} a_{e} a_{f}}{a_{e e}} t_{f}^{*} .
\end{aligned}
$$

We now investigate how an improvement in telecommunications technology affects the time spent on either mode of interaction. First, the effect on the optimal time spent of electronic communications is as follows:

$$
\frac{\partial t_{e}^{*}}{\partial a_{e}}=\frac{b^{2}\left(a_{e} a_{f} a_{e f}\right)\left(a_{f} a_{e f} b t_{e}\right)+b^{2} a_{f f}\left(1-a_{f} a_{e f} b t_{f}\right)}{|H|}
$$

Since the first term in the above expression is positive, the second term is positive by the FOCs, and the Hessian determinant is also positive by the second order conditions, it follows that $\frac{\partial t_{e}^{*}}{\partial a_{e}}>0$, i.e., an improvement in electronic communications increases the time spent on electronic interactions, since now it's easier and faster to communicate electronically.

Second, the effect of improvements in telecommunications technology on the optimal time spent on faceto-face interactions is as follows:

$$
\frac{\partial t_{f}^{*}}{\partial a_{e}}=\frac{-b\left(a_{e} a_{f} a_{e f}\right)\left(1-a_{f} a_{e f} b t_{f}\right)-b a_{e e}\left(a_{f} a_{e f} b t_{e}\right)}{|H|}
$$

Since the first term in the above expression is negative by the FOCs, the second term is negative and the Hessian determinant is also positive by the second order conditions, it follows that $\frac{\partial t_{f}^{*}}{\partial a_{e}}<0$, i.e., an improvement in electronic communications decreases the time spent on face-to-face interactions.

In addition the following comparative static result is needed to determine the effect of advances in information technology on city size,

$$
\frac{\partial t_{e}^{*}}{\partial b}=-\frac{c a_{e f} a_{e} a_{f}}{|H|}<0
$$


Result (10) implies that an increase in the productive time of face-to-face contact will decrease the time spent on electronic contact, as people substitute electronic with face-to-face contact.

General Model II: (Face-to-face interactions and electronic communications are complements): The payoff from the relationships is now given by the following function,

$V=a_{e} t_{e}+a_{f} b t f-\frac{1}{2} a_{e e} t^{2}-\frac{1}{2} a_{f f} b^{2} t_{f}^{2}+a_{e} a_{f} a_{e f} e_{e}^{b t} f{ }^{4}$

Note that $\frac{\partial^{2} V}{\partial t_{e} \partial b t f}=a_{e} a_{f} a_{e f}>0$, implying that face-to-face interactions and electronic communications are complements. As in model I, the individual maximizes her net payoff form the relationships. Namely, she is faced with the following problem:

$\operatorname{Max} \Pi=a_{e} t_{e}+a_{f} b t_{f}-\frac{1}{2} a_{e e} t_{e}^{2}-\frac{1}{2} a f f b^{2} t_{f}^{2}+a_{e} a_{f} a_{e f} t_{e}^{b t} f-c\left(t_{e}+t_{f}\right)$

The first order conditions (FOCs) for payoff maximization are

$$
\begin{aligned}
& \frac{\partial \Pi}{\partial t_{e}}=a_{e}-a_{e e} t_{e}+a_{e} a_{f} a_{e f} b t_{f}-c=0 \\
& \Rightarrow\left(1+a_{f} a_{e f} b t_{f}\right)=\frac{c+a_{e e} t_{e}}{a_{e}}, \\
& \frac{\partial \Pi}{\partial t_{f}}=b a_{f}-a_{f f} b^{2} t_{f}+a_{e} a_{f} a_{e f} b t_{e}-c=0 \\
& \Rightarrow\left(1+a_{e} a_{e f} t_{e}\right)=\frac{c+a_{f f} b^{2} t_{f}}{b a_{f}} .
\end{aligned}
$$

As in model I, both first order conditions imply that the marginal payoff from either mode of interaction must be equal with the marginal cost, i.e., the opportunity cost of time.

Following similar analysis as in the general model I, the following optimal solutions $t_{e}^{*}$ and $t_{f}^{*}$ are obtained:

$$
t_{f}^{*}=\frac{b a_{e e} a_{f}+b a_{e f} a_{f} a_{e}^{2}-b c a_{e f} a_{e} a_{f}-c a_{e e}}{|H|},
$$

\footnotetext{
${ }^{4}$ The difference between the payoff function (11) and the payoff function in Panayides (2001) is that $a_{e}$ and $a_{f}$ affect both $t_{e}$ and $t_{f}$, which implies that if the two modes of interaction are complements, both partial derivatives $\frac{\partial^{2} V}{\partial t_{e} \partial a_{f}}$ and $\frac{\partial^{2} V}{\partial b t_{f} \partial a_{e}}$ are not zero anymore but positive.
} 


$$
t_{e}^{*}=\frac{a_{e}-c}{a_{e e}}+\frac{b a_{e f} a_{e} a_{f}}{a_{e e}} t_{f}^{*} .
$$

We now investigate how an improvement in telecommunications technology affects the time spent on either mode of interaction. These effects are:

$$
\begin{aligned}
& \frac{\partial t_{e}^{*}}{\partial a_{e}}=\frac{b^{2}\left(a_{e} a_{f} a_{e f}\right)\left(a_{f} a_{e f} b t_{e}\right)+b^{2} a_{f f}\left(1+a_{f} a_{e f} b t_{f}\right)}{|H|}>0 . \\
& \frac{\partial t_{f}^{*}}{\partial a_{e}}=\frac{b\left(a_{e} a_{f} a_{e f}\right)\left(1+a_{f} a_{e f} b t_{f}\right)+b a_{e e}\left(a_{f} a_{e f} b t_{e}\right)}{|H|}>0 .
\end{aligned}
$$

These results imply that, if face-to-face interactions and electronic communications are complements, an improvement in electronic communications increases the time spent on both modes of interactions. The reason for this result is that an improvement in electronic communications unambiguously increases the time spent on electronic communications since, as stated above, now it's easier and faster to communicate electronically, result (17). Hence, if electronic communications and face-to-face interactions are complements, then improvements in communications technology will also increase the time spent on face-to-face interactions, result (18).

In addition the following comparative static result is needed to determine the effect of advances in information technology on city size,

$$
\frac{\partial t_{e}^{*}}{\partial b}=\frac{c a_{e f} a_{e} a_{f}}{|H|}>0 .
$$

Result (19) implies that an increase in the productive time of face-to-face contact will increase the time spent on electronic contact.

From both general models we can conclude that improvements in communications technology unambiguously increases the time spent on electronic communications. However, improvements in communications technology increase the time spent on face-to-face interactions only if face-to-face interactions and electronic interactions are complements. If they are substitutes then improvements in communications technology results in reduction of time spent on face-to-face interactions.

\section{The Urban Model}

In this section the payoff model developed above is placed within a spatial framework to examine how changes in electronic communications affects the location choice of a resident. A representative agent may work in the city center or outside the city, which is referred to as the hinterland. The differences between the city and the hinterland are commuting cost and rent premiums for housing, which are denoted by $\mathrm{HN}$, where $\mathrm{N}$ is the population of the city. The other difference between the two locations is that, we assume that the city facilities face-to-face interactions, i.e., the fraction of face-to-face interactions that is productive is higher in the city than in the hinterland.

As in the case of the payoff model, similarly in the urban model, first, the quadratic function is so constructed that face-to-face interactions and electronic communications are substitutes (urban model I), and then such that face-to-face interactions and electronic communications are complements (urban model II).

Urban Model I (Face-to-face interactions and electronic communications are substitutes): The payoff and 
net payoff functions for a resident are given as follows,

$$
\begin{aligned}
& \Pi=a_{e} t_{e}+a_{f} b t_{f}-\frac{1}{2} a_{e e} t_{e}^{2}-\frac{1}{2} a_{f f} b^{2} t_{f}^{2}-a_{e} a_{f} a_{e f} e_{e}^{b t} f \\
& \Pi=a_{e} t_{e}+a_{f} b t_{f}-\frac{1}{2} a_{e e} t_{e}^{2}-\frac{1}{2} a_{f f} b^{2} t_{f}^{2}-a_{e} a_{f} a_{e f} t_{e} b t_{f}-c\left(t_{e}+t_{f}\right)-H N-R .^{5}
\end{aligned}
$$

The focus here is to examine how an improvement in electronic communications affects the location choice of a resident. Since except for the congestion cost differences, the city and the hinterland differ in the productivity of face-to-face communication, in order to examine this effect we need the effect of $b$ on $\frac{\partial V}{\partial a_{e}}$. differentiating the payoff function (20) with respect to $a_{e}$ yields

$$
\frac{\partial V}{\partial a_{e}}=t_{e}-a_{f} a_{e f} t_{e} b t_{f}
$$

Differentiating (22) with respect to $b$ yields

$$
\begin{aligned}
& \frac{\partial^{2} V}{\partial a_{e} \partial b}=\frac{\partial t_{e}}{\partial b}-a_{f} a_{e f} b t_{f}\left(\frac{\partial t_{e}}{\partial b}\right)-a_{f} a_{e f} t_{e}\left(\frac{\partial b t_{f}}{\partial b}\right) \\
& =\frac{\partial t_{e}}{\partial b}\left[1-a_{f} a_{e f} b t_{f}\right]-a_{f} a_{e f} t_{e}\left(\frac{\partial b t_{f}}{\partial b}\right)<0 .
\end{aligned}
$$

The expression (23) is negative because if face-to-face interactions and electronic communications are substitutes then $\frac{\partial t_{e}}{\partial b}<0$ from (10), the term $\left\lfloor 1-a_{f} a_{e f} b t_{f}\right\rfloor>0$ from FOCs (3) and $\frac{\partial b t_{f}}{\partial b}>0$. Since by assumption the fraction of face-to-face interactions that is productive is higher in the city than in the hinterland, i.e., $b$ is higher in the city than in the hinterland, result (23) implies that an improvement in electronic communications increases the payoff less for city residents than for those in the hinterland. Hence more people will move out of the city, to avoid the higher living and commuting costs, and thus city population will fall. In other words, if face-toface interactions and electronic communications are substitutes, an improvement in electronic communications decreases city size.

Urban Model II (Face-to-face interactions and electronic communications are complements): The payoff and net payoff functions for a resident are given as follows,

$$
\begin{aligned}
& \Pi=a_{e} t_{e}+a_{f} b t_{f}-\frac{1}{2} a_{e e} t_{e}^{2}-\frac{1}{2} a f f b^{2} t_{f}^{2}+a_{e} a_{f} a_{e f} e_{e}^{b t} f \\
& \Pi=a_{e} t_{e}+a_{f} b t_{f}-\frac{1}{2} a_{e e} t_{e}^{2}-\frac{1}{2} a_{f f} b^{2} t_{f}^{2}+a_{e} a_{f} a_{e f} t_{e}^{b t} f-c\left(t_{e}+t_{f}\right)-H N-R .
\end{aligned}
$$

\footnotetext{
${ }^{5}$ For a hinterland resident there are no costs HN. This does not affect any of the results since HN is a constant. R denotes the agricultural rent.
} 
Following similar analysis as in Urban Model I, since the difference between the city and the hinterland is only the productivity of face-to-face communication, in order to examine how improvements in electronic communication affects location decisions by residents, we need the effect of $b$ on $\frac{\partial V}{\partial a_{e}}$. First, differentiating the payoff function (24) with respect to $a_{e}$ yields

$$
\frac{\partial V}{\partial a_{e}}=t_{e}+a_{f} a_{e f} t_{e} b t_{f}
$$

Differentiating (26) with respect to $b$ yields

$$
\begin{aligned}
& \frac{\partial^{2} V}{\partial a_{e} \partial b}=\frac{\partial t_{e}}{\partial b}+a_{f} a_{e f} b t_{f}\left(\frac{\partial t_{e}}{\partial b}\right)+a_{f} a_{e f} t_{e}\left(\frac{\partial b t_{f}}{\partial b}\right) \\
& =\frac{\partial t_{e}}{\partial b}\left[1+a_{f} a_{e f} b t_{f}\right]+a_{f} a_{e f} t_{e}\left(\frac{\partial b t_{f}}{\partial b}\right)>0 .
\end{aligned}
$$

The expression (27) is positive because if face-to-face interactions and electronic communications are complements then $\frac{\partial t_{e}}{\partial b}>0$ from (19), the term $\left\lfloor 1+a_{f} a_{e f} b t_{f}\right\rfloor>0$ from FOCs (13) and $\frac{\partial b t_{f}}{\partial b}>0$. Following the same logic as above, since by assumption $b$ is higher in the city than in the hinterland, result (27) implies that an improvement in electronic communications increases the payoff more for city residents than for residents of the hinterland. Hence more people will move into the city and thus city population will rise. In other words, if face-toface interactions and electronic communications are complements, an improvement in electronic communications increases city size.

From both urban models we can conclude that an improvement in electronic communications may increase or decrease city size. Particularly, if face-to-face interactions and electronic communications are complements then improvements in telecommunications technology will increase city size, while if they are substitutes then improvements in telecommunications technology will decrease city size.

\section{Conclusion}

In this paper we developed a model using a specific quadratic function to examine the interdependence between face-to-face interactions and electronic communications. The general model shows that improvements in communications technology increases the time spent on electronic interactions, but the effect on face-to-face interactions is ambiguous. Specifically, improvements in communications technology increase the time spent on face-toface interactions only if the two modes of interaction are complements. The placement of the model within an urban framework shows that if the face-to-face interactions and electronic communications are complements then improvements in telecommunications technology increases city size.

\section{References}

1. Gaspar and Glaeser (1998), "Information Technology and the Future of Cities", Journal of Urban Economics, 43, 136-156.

2. Knoke, K. (1996), Bold New World: The Essential Road Map to the Twenty-First Century, Kodansha, New York.

3. Naisbitt, R. (1995), The Global Paradox, Avon Books, New York. 
4. $\quad$ Negroponte, N. (1995), Being digital, Vintage Books, New York.

5. Panayides, A. (2001), "The effects of Improvements in Information Technology on Growth of Cities", Mimeo.

6. Toffler, A. (1980), The Third Wave, Bantam Books, New York.

Notes 